\title{
Laparoscopic Cholecystectomy Intervention in Rats with Severe Acute Pancreatitis Delays Apoptosis of Myocardial Cells
}

\author{
X. YE, M. WANG, X. FANG, CHUNLIAN TU, L. WANG* \\ Department of ICU, Shanghai Yangpu District Shidong Hospital, Shanghai, 200438, China
}

Ye et al.: Laparoscopic Cholecystectomy Intervention Delays Myocardial Apoptosis

\begin{abstract}
In order to study the mechanism of laparoscopic cholecystectomy intervention in macrophages of rats with severe acute pancreatitis to delay apoptosis of cardiac myocytes, Wistar rats were chosen and divided into 3 groups. In addition, the related indices and protein expression in rats were observed at 2, 8, 16 and $24 \mathrm{~h}$ for each component and caspase-3 and bcl-2 levels in myocardium are detected to study the effect of laparoscopic cholecystectomy intervention. Results indicated that there were no obvious symptoms in control rats, while the rats in intervention group have the same symptoms as the experimental group. White blood cells of the 3 groups of rats, when compared it was found that the white blood cells in the experimental group decrease, followed by the intervention group but there is no significant change in the control group. Morphological examination of myocardial tissue from the 3 groups of rats indicated that there was no significant change in myocardial cells in the control group, while piles of blood cells were found between myocardial cells in the experimental group with obvious nuclear deformation. There were patches of blood between myocardial cells in the intervention group, without any significant changes in nucleus. Among the changes in the content of tumour necrosis factor- $\alpha$ and apoptosis of myocardial tissue, it is found that the tumour necrosis factor- $\alpha$ content and apoptotic phenomena were most significant in the experimental group and there was a significant correlation between the experimental group and the control group. After laparoscopic cholecystectomy, it was found that the content of tumour necrosis factor- $\alpha$ in myocardial tissue decreased and apoptotic phenomena were alleviated, which was significantly different from the experimental group $(p<0.01)$. Western blot results indicated that expression of caspase-3 and bcl-2 in myocardial tissue were the highest in the experimental group, followed by the intervention group and the lowest in the control group. It was also found that the change of tumour necrosis factor- $\alpha$ was synchronous and positively correlated with the change of apoptotic index of myocardial cells. Therefore, it could be concluded that laparoscopic cholecystectomy could be used to intervene in severe acute pancreatitis to delay the apoptosis of myocardial cells in rats.
\end{abstract}

Key words: Acute pancreatitis, LC, TNF-a, myocardial tissue

Acute pancreatitis is one of the most common diseases in China. The cause of this inflammation is the activation of pancreatic enzyme in human pancreas, which leads to the occurrence of digestion, oedema, haemorrhage or necrosis in human body ${ }^{[1,2]}$. For patients, there is often acute epigastric pain, nausea or vomiting, and the symptoms of acute pancreatitis vary in severity. For those with mild symptoms, pancreatic oedema may occur in vivo, but the clinical prognosis is good. Most of the cases are self-limited, which is mild acute pancreatitis ${ }^{[3]}$. Severe acute pancreatitis is often associated with pancreatic haemorrhage and necrosis. There are often secondary infections and abdominal inflammation and shock. The probability of death is very high. In the past decade, more than 2980 studies on cardiac dysfunction caused by severe acute pancreatitis have been published in $\mathrm{China}^{[4]}$. Therefore, severe acute pancreatitis is important for the occurrence and development of cardiac dysfunction. When laparoscopic cholecystectomy (LC) technology has not been widely used in hospitals, abdominal cholecystectomy for acute pancreatitis is an effective way to reduce the recurrence of the disease. However, this operation also has its drawbacks. Postoperative

*Address for correspondence

E-mail: wanglinlinll0895@126.com

Special Issue 6, 2020 
recovery is slow and there may be many complications, which are not easily accepted from the perspective of patients and their families. Therefore, in China where TCM technology is developed, doctors often use conservative treatment, which may lead to repeated attacks and eventually aggravate the disease gradually. The cost will not be small ${ }^{[5-7]}$ and therefore, the clinical study of severe acute pancreatitis has become the focus of attention of scholars.

Tumour necrosis factor- $\alpha$ (TNF- $\alpha)$ is a cytokine with many excellent biological characteristics produced by macrophages in the immune system in the activated state. In human body, TNF- $\alpha$ may impair cardiac function, leading to apoptosis of cardiac myocytes, and some studies have found that TNF- $\alpha$ levels may be related to a variety of cardiac disease ${ }^{[8]}$. In the process of cell apoptosis, it participates in the formation of cell scaffolds, reduction of fixed cell size and the concentration and breakage of DNA in nucleus and chromosomes. In addition, cell membranes display blistering and crushing phenomena. At the same time endonucleases are activated. Membranous apoptotic bodies also appear, which indicate cell apoptosis ${ }^{[9,10]}$. The apoptosis of cells refer to programmed cell death rather than random occurrence, which is accomplished by the cooperation of cysteine protease and its family known as caspase ${ }^{[1-13]}$. It is observed that chemotherapeutic drugs used currently act by the mechanism of cell transformation to remove cancer cells. At the same time, cell apoptosis can also lead to drug resistance ${ }^{[14]}$. Some studies reported that in patients with heart failure, the content of TNF- $\alpha$ in myocardial tissue and blood would increase significantly. TNF- $\alpha$ has a negative effect on human muscle strength, which could also participate in remodelling of myocardium after heart failure by inducing cardiomyocyte apoptosis. Therefore, TNF- $\alpha$ can be used as an independent risk factor to predict mortality in patients with heart failure ${ }^{[15]}$.

In conclusion, although there are many related studies on molecular biological therapy of severe acute pancreatitis, the mechanism of cardiomyocyte apoptosis after LC intervention has not been understood through continuous exploration of severe acute pancreatitis. Therefore, in this study, Wistar rats are selected as a test system, which were divided into 3 groups to observe the related indicators and protein expression to analyse the effect of LC intervention on myocardium in rats with severe acute pancreatitis, so as to provide new ideas for the treatment of acute pancreatitis in the future. Therefore, this study has great significance.

\section{MATERIALS AND METHODS}

\section{Animals:}

Ninety six male Wistar rats (180-220 g) were purchased from Beijing Weitong Lihua Laboratory Animal Technology Co., Ltd., China, which meet the national animal standard. Animal disposal and experimental procedures are in line with the national standards for laboratory animals. All animal experiment programs have been approved by the Ethic Committee of Shanghai Yangpu District Shidong Hospital.

\section{Animal model preparation and grouping:}

A rat model of severe acute pancreatitis was established by performing LC. Firstly, a special guidance tube was inserted into the peritoneal cavity and 2-5 1 of carbon dioxide was injected. After a certain pressure is reached, four small holes of $0.5-1.5 \mathrm{~cm}$ were made in the abdomen. The structure of the gallbladder triangle was dissected. The gallbladder duct and the gallbladder artery were cut off and clamped. Then, the entire gallbladder was removed.

Thirty-two rats were divided into the experimental group (LC). Severe acute pancreatitis model was established by LC. The hearts were removed at 2, 8, 16 and $24 \mathrm{~h}$ after anaesthesia (8 rats at each time point). In the control group, 32 rats were anesthetized and the hearts were removed at 2, 8, 16 and $24 \mathrm{~h}(8$ rats at each time point), except that LC was not performed. Thirty-two rats in the intervention group were injected intraperitoneally with $3 \mathrm{ml}$ of antiTNF $\alpha-\mathrm{mAb}$ $0.4 \mathrm{mg} / \mathrm{kg}$ dissolved in saline $30 \mathrm{~min}$ before operation. The other groups were injected with normal saline (Shanghai Xinyu Biotechnology China) and hearts were taken out directly after $2,8,16$ and $24 \mathrm{~h}$ (8 at each time point).

\section{Sample collection and processing:}

After anaesthesia, rats are routinely disinfected abdominal skin, abdominal cavity is opened and abdominal aorta is separated. Five millilitres of abdominal aortic blood was collected with a $10 \mathrm{ml}$ syringe and blood collection needle. Two millilitres of arterial blood was taken in a sterile test tube for routine blood determination. The remaining $3 \mathrm{ml}$ is placed in a dry glass tube for $10 \mathrm{~min}$, and centrifuged at $8^{\circ}, 4000 \mathrm{rpm}$ for $15 \mathrm{~min}$. Then, serum was separated. After separate packing, it was stored at $-25^{\circ}$. Sterile procedures were strictly followed to extract the heart and the ventricles and atria were separated. The remaining left ventricular 
myocardium was cut and stained with $\mathrm{HE}$ in alcoholic formaldehyde. Wax blocks and pathological sections were prepared. The remaining part was stored in liquid nitrogen in cryopreservation tube and stored in a $-85^{\circ}$ deep freezer.

\section{Observation indicators:}

White blood cells in serum were measured in a white blood cell analyser (Beckmann Kurt Company, USA, C.T5iff). HE staining was used to observe pathological changes of myocardial tissue. The myocardial tissue was extracted and fixed with $10 \%$ formaldehyde solution, and then embedded in paraffin. The slice thickness was kept at 6-7 $\mu \mathrm{m}$. After dewaxing, HE staining was performed routinely and the sections were observed under a microscope (TCS SP8, Leica, Germany). The detection method of TNF- $\alpha$ in myocardial tissue was to extract myocardial tissue frozen at $-85^{\circ}$, weighed 150 $\mathrm{g}, 3 \mathrm{ml}$ saline was added to prepare the homogenate in an ultrasonic homogenizer, centrifuged at $1500 \mathrm{rpm}$ for $25 \mathrm{~min}$ at $8^{\circ}$ and the supernatant was collected. The sensitivity of rat TNF- $\alpha$ ELISA kit used was 5 pg/ $\mathrm{ml}$. determination was done strictly according to the instructions of the kit. The standard curve was plotted with OD as ordinate and standard concentration as abscissa. According to the OD value of the experimental sample, the corresponding concentration can be found in the chart. Rat TNF- $\alpha(\mathrm{pg} / \mathrm{ml})=$ the concentrations found on standard curves $\times$ dilution multiple/50 000 .

\section{Detection of cardiomyocyte apoptosis:}

The procedure according to the instructions of the kit was followed. Rat hearts were fixed with $10 \%$ formaldehyde, routinely embedded in paraffin, sliced, dewaxed, and hydrated with ethanol of different concentration gradients. It is digested by pepsin for $15 \mathrm{~min}$ and washed twice with PBS for $10 \mathrm{~min}$ in a wet box $\left(37^{\circ}\right)$. Hydrogen peroxide-methanol solution $(0.3 \%)$ was added to block endogenous peroxidase. Kept aside for $20 \mathrm{~min}$, washed twice with PBS for 10 min. TUNEL reaction liquid was added. It was placed in a refrigerator overnight in a wet box $\left(8^{\circ}\right)$. PBS was used to wash 3 times for 10 min each time. StreptavidinHRP working fluid was added and allowed to react for $40 \mathrm{~min}$ at $37^{\circ}$. Washed twice with PBS for $10 \mathrm{~min}$. Aminobenzidine (DAB) was used to dye for $20 \mathrm{~min}$, and then re-dyed with hematoxylin. Tree gum is used as a seal and observed under a microscope. Under light microscopy, the apoptotic positive nuclei were yellow stained. Four high power microscopic fields (X400) were randomly selected from the same section to calculate the proportion of positive cells and the average value is the apoptotic index (AI) of cardiomyocyte apoptosis.

\section{Detection of Caspase-3 in myocardial tissue:}

Western blot is used to detect the expression of Caspase-3 in myocardium. Firstly, myocardial tissue was lysed and myocardial tissue protein was extracted. The total protein content of myocardial tissue was determined by Bradford method. Sodium dodecyl sulfate-polyacrylamide gel electrophoresis was used to separate proteins with different molecular weights. The sample is equivalent to electrophoresis in $12 \%$ of polyacrylamide gel. Electron transfer membranes are used to transfer proteins to cellulose nitrate membranes. Coomassie Bright Blue is used as a dye. After the metastasis effect is observed, elution is performed. TBST (Hangzhou Beiwa Medical Technology Co., Ltd., China) buffer containing $5 \%$ skimmed milk was used for overnight closure. Goat antirat caspase-3 primary antibody (1:200) was added for hybridization, and then rabbit antigoat IgG secondary antibody labelled with horseradish peroxidase (1:500, Sigma, USA) was used for immune response. DAB (Hangzhou Beiwo Medical Technology Co., Ltd., China) was used for colour rendering. The results were analysed in the image analysis system Scion Corporation.

\section{Detection of bcl-2 in myocardial tissue:}

Western blot was used to detect the expression of bcl-2 protein in myocardium (Shanghai Xinyu Biotechnology Co., Ltd., China). The steps are the same as above. Goat antirat bcl-2 primary antibody (1:400) was added for hybridization, and then rabbit antigoat IgG secondary antibody labelled with horseradish peroxidase (1:500) was used for immune response and $\mathrm{DAB}$ was used for colour rendering. The results were analysed in the image analysis system Scion Corporation.

\section{Statistical methods:}

SPSS20.0 statistical software is used for statistical analysis. The counting data are expressed by percentage and composition ratio by descriptive statistical method, and accorded with the normal distribution of the counting data. Chi-square test is used, and $\mathrm{p}<0.05$ has statistical significance. Significance $t$ test is used for the measurement data with normal distribution, which is expressed as mean \pm standard deviation (SD). $\mathrm{P}<0.05$ has statistical significance. 


\section{RESULTS AND DISCUSSION}

After awakening, the rats in the experimental group were depressed and have no vision. After $2 \mathrm{~h}$, the pathological condition became more and more serious, such as sleepiness, obvious decrease of daytime activity, no desire to drink water, no appetite, faster respiratory rate, and aversion to cold. Sometimes, a large number of white blood cells appeared in urine. There was diarrhoea, eye droppings and other phenomena. Before death, the animals experienced breathlessness, laborious breathing and trembling limbs. The above changes were most obvious at $24 \mathrm{~h}$. In the control group, rats were less active but returned to normal activity with in a period of time. The rats in the intervention group had the same symptoms as those in the experimental group, but the symptoms are not as severe as those in the experimental group.

In the experimental group, the number of white blood cells in 4 rats decreased by $45 \%$ in $2 \mathrm{~h}$ compared to that of the control group and the rest four are only three times of the average of the control group. The number of white blood cells in 6 rats decreased by $45 \%$ at $12 \mathrm{~h}$ compared to that of the control group, while the number of white blood cells in the remaining 2 rats was two times higher than that in the control group. The number
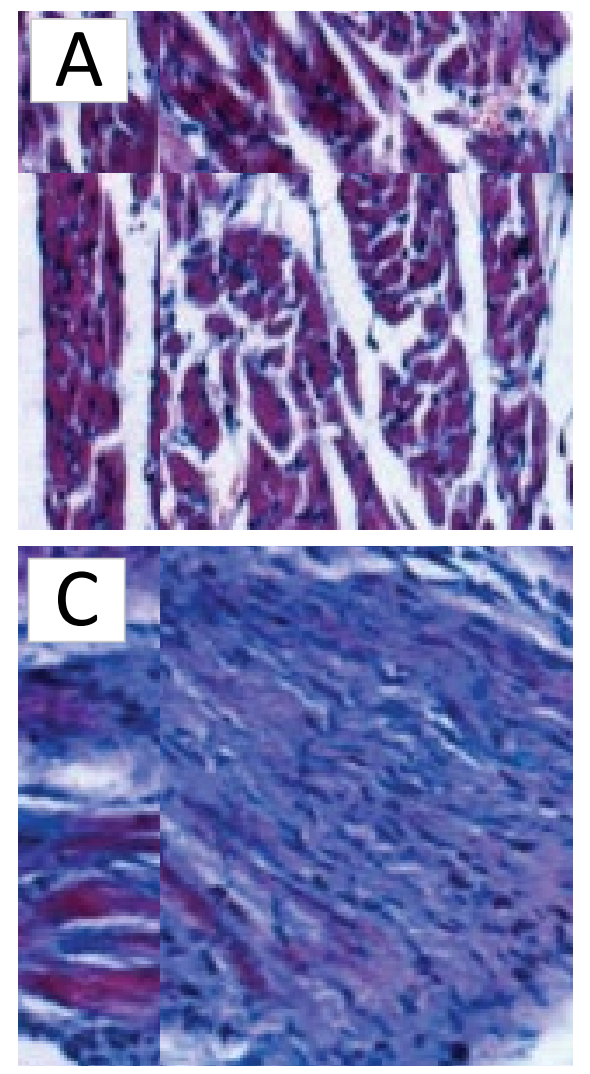

Fig. 1: Morphological changes in myocardial tissue in rats

A. 3 h myocardiogram of control group, B. 24-h myocardiogram of experimental group, C. 36-h myocardiogram of experimental group and D. 24-h myocardiogram of intervention group of white blood cells in 5 rats at $16 \mathrm{~h}$ was two times higher than that in the control group, and 3 were less than $45 \%$ of the control group. At $24 \mathrm{~h}$, the white blood cells in 8 blood samples were less than $45 \%$ of those in the control group, and the decrease is most obvious at $24 \mathrm{~h}$.

It can be observed from fig. 1A that no obvious pathological changes were found in the control group, while in the experimental group (fig. 1B, C), myocardial tissue was found disordered, blood vessels were congested and bleeding and patches of blood cells were seen between myocardial cells, especially at 24 h. Myocardial cells were congested, swollen or shrunk due to lack of water. The nucleus was deformed. From fig. 1D, it was evident that the myocardial tissue of intervention group was disordered with bleeding spots but there were no obvious changes in the nuclei.

The changes in TNF- $\alpha$ levels in myocardial tissue of rats after LC are shown in Table 1 and fig. 2. The results of ELISA showed that compared to the control group, there were significant differences in the experimental group at each time point $(p<0.01)$. There were also significant differences between the intervention group and the experimental group at each time point $(\mathrm{p}<0.01)$. The content of TNF- $\alpha$ in the rats of experimental group
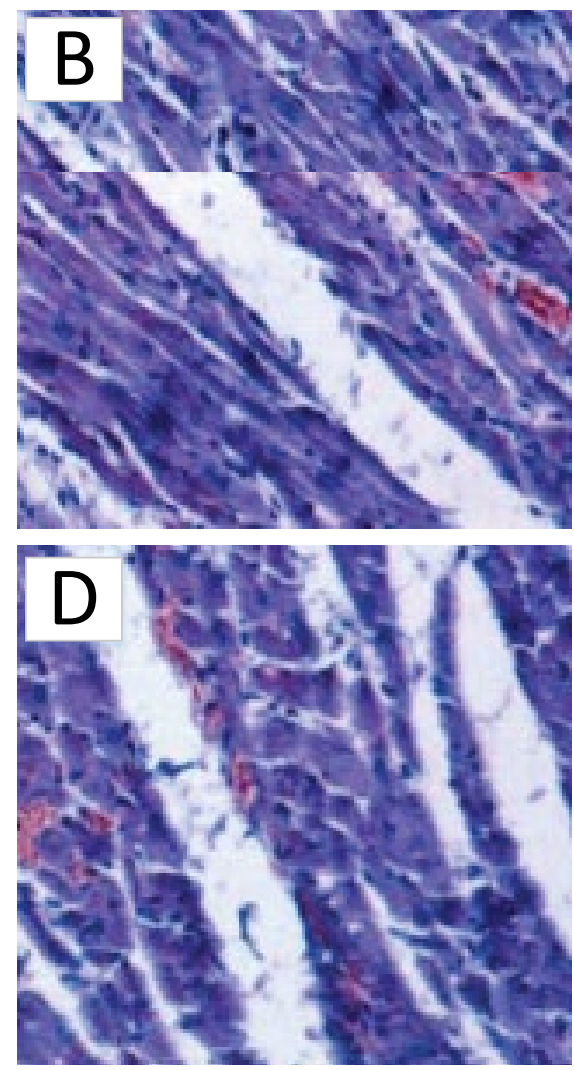
increased gradually but significantly with time $(\mathrm{p}<0.05)$. Therefore, it could be concluded that acute pancreatitis increased the content of TNF- $\alpha$ in rats, and intervention could reduce the level of TNF- $\alpha$ in myocardial tissue.

The changes in cardiomyocyte $\mathrm{AI}$ in rats after LC are shown in Table 2. TUNEL test showed that there were significant differences between the experimental group and the control group at each time point $(\mathrm{p}<0.01)$. There were also significant differences between the intervention group and the experimental group at each time point $(\mathrm{p}<0.01)$, and the apoptosis of myocardial cells in the experimental group increased with time. Acute pancreatitis could cause apoptosis of rat cardiac myocytes, which increased with time and intervention slowed down the apoptotic level of rat cardiac myocytes (fig. 3).

From fig. 4, it was evident that the expression of caspase-3 in myocardial tissue increased in the experimental group. However the expression was lower in the control group compared to the experimental

\section{TABLE 1: COMPARISON OF TNF- $\alpha$ LEVEL IN THE MYOCARDIUM OF THREE GROUPS OF RATS AT DIFFERENT TIME POINTS}

\begin{tabular}{cccc}
\hline Time (h) & Control group & $\begin{array}{c}\text { Experimental } \\
\text { group }\end{array}$ & $\begin{array}{c}\text { Intervention } \\
\text { group }\end{array}$ \\
\hline 2 & $411.00 \pm 12.330$ & $485.63 \pm 31.722^{\#}$ & $369.50 \pm 39.688^{*}$ \\
8 & $422.32 \pm 4.988$ & $578.84 \pm 14.661^{\#}$ & $439.78 \pm 20.009^{*}$ \\
16 & $428.12 \pm 20.899$ & $695.34 \pm 43.002^{\# b c}$ & $490.11 \pm 43.892^{*}$ \\
24 & $450.88 \pm 19.023$ & $737.89 \pm 48.767^{\# b c}$ & $533.73 \pm 26.433^{*}$
\end{tabular}

TNF- $\alpha$ levels were expressed as $\mathrm{pg} / \mathrm{ml}$. "Significant difference compared to control group $(p<0.01)$. *Significant difference compared to the experimental group $(P<0.01)$. 'bignificant difference compared to the experimental group at $2 \mathrm{~h}(\mathrm{P}<0.01)$. cSignificant difference compared to the experimental group at $16 \mathrm{~h}$ $(p<0.01)$. There was no significant difference between $8 \mathrm{~h}$ and $24 \mathrm{~h}$ in the experimental group

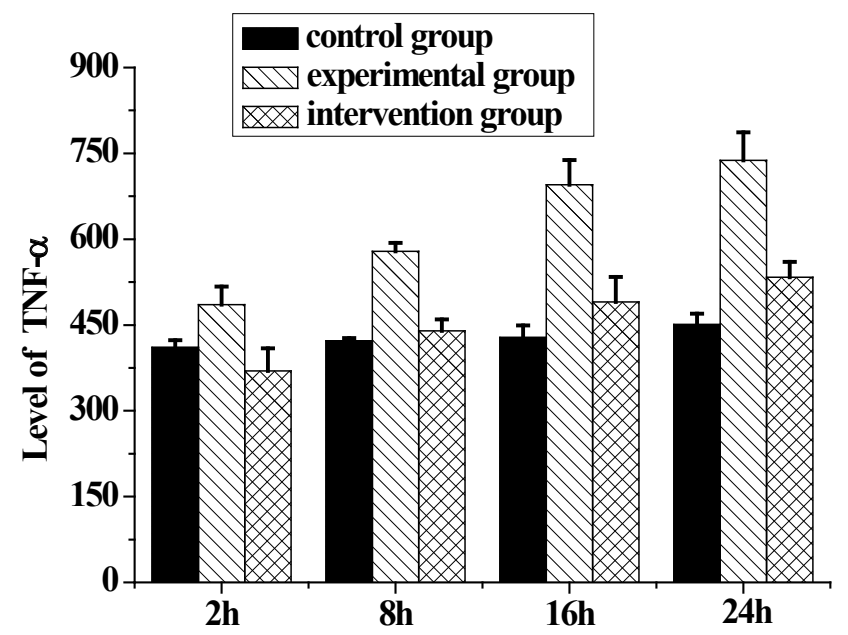

Fig. 2: TNF- $\alpha$ levels in myocardium detected by ELISA

(a) Control group, ( $)$ experimental group, (网) intervention group

Special Issue 6, 2020

Indian Journal of Pharmaceutical Sciences

35
TABLE 2: COMPARISON OF APOPTOTIC INDEX OF MYOCARDIAL TISSUE AT DIFFERENT TIME POINTS IN THREE GROUPS OF RATS

\begin{tabular}{lccc}
\hline $\begin{array}{l}\text { Time } \\
\text { (h) }\end{array}$ & Control group & $\begin{array}{c}\text { Experimental } \\
\text { group }\end{array}$ & $\begin{array}{c}\text { Intervention } \\
\text { group }\end{array}$ \\
\hline 2 & $0.879 \pm 0.013$ & $9.593 \pm 1.178^{\#}$ & $3.345 \pm 0.987^{*}$ \\
8 & $1.234 \pm 0.133$ & $25.354 \pm 5.123^{\#}$ & $13.344 \pm 1.467^{*}$ \\
16 & $2.346 \pm 0.487$ & $39.786 \pm 4.675^{\text {\#c }}$ & $20.785 \pm 1.564^{*}$ \\
24 & $4.657 \pm 0.0786$ & $43.762 \pm 2.487^{\# \text { bc }}$ & $24.565 \pm 1.987^{*}$ \\
\hline
\end{tabular}

Apoptotic index was expressed as a '\%'. "Significant difference compared to control group $(p<0.01)$. *Significant difference compared to the experimental group $(p<0.01)$. b Significant difference compared to the experimental group at $2 \mathrm{~h}(\mathrm{P}<0.01)$. cSignificant difference compared to the experimental group at $8 \mathrm{~h}$ $(p<0.01)$. There was no significant difference between 16 and $24 \mathrm{~h}$ in the experimental group

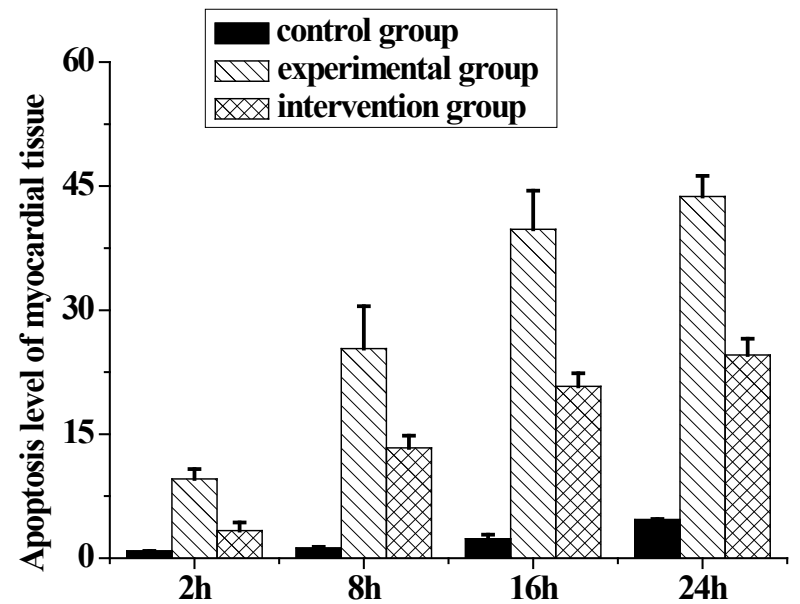

Fig. 3: Apoptotic index (AI) detected by TUNEL

(a) Control group, ( ) experimental group, ( ( ) intervention group

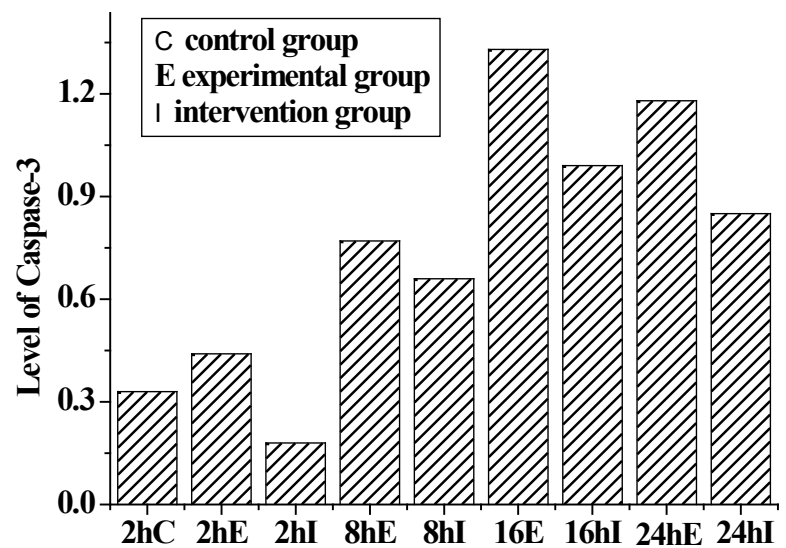

Fig. 4: Changes of Caspase-3 in myocardial tissue

$\mathrm{C}$ : Control group, E: experimental group, I: intervention group

group and intervention reduced the expression. Fig. 5, shows the expression of bcl-2 in myocardial tissue as detected by Western blot. Expression increased in the experimental group, expression in the control group was lower than the experimental group, and in the intervention group also the expression was lower. Fig. 6 shows changes in TNF- $\alpha$ were synchronous with 


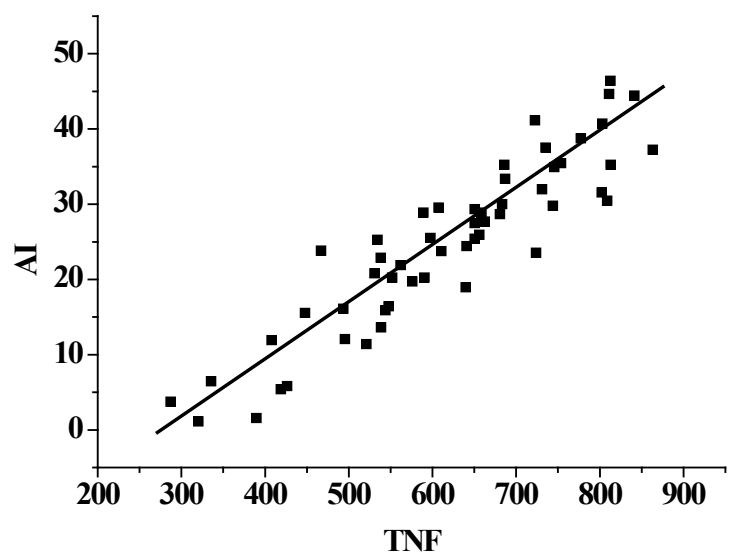

Fig. 5: Changes of bcl-2 in myocardium $\mathrm{C}$ : Control group, E: experimental group, I: intervention group

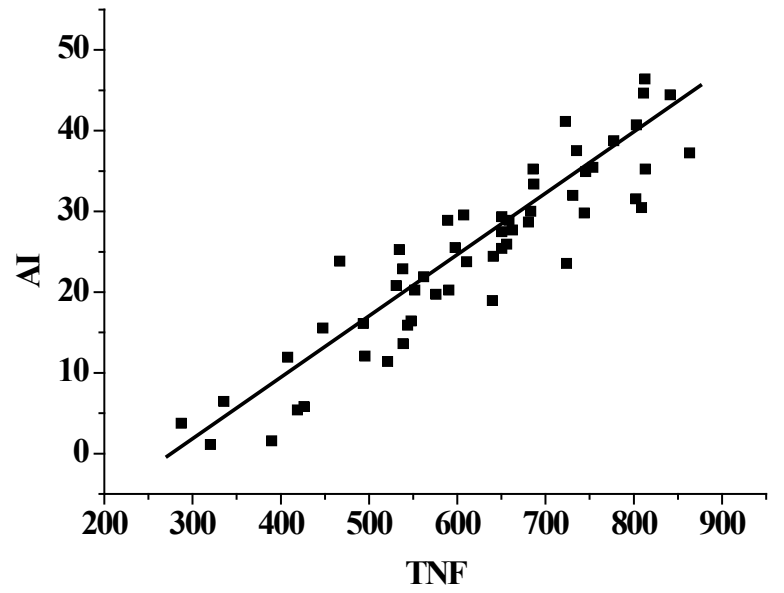

Fig. 6: Scatter plot of changes of TNF and cardiac apoptotic index

the changes of cardiac $\mathrm{AI}(\mathrm{r}=0.897)$, with the regression Eqn. being Y $(\mathrm{AI})=-41.985+0.121 \mathrm{X}(\mathrm{TNF}-\alpha)$.

In order to study the mechanism of LC intervention in rats with severe acute pancreatitis to delay apoptosis of cardiac myocytes, Wistar rats were selected and divided into 3 groups. In addition, the related indices and protein expression in rats were observed at 2, 8, 16 and $24 \mathrm{~h}$ for each component, and caspase-3 (cysteinyl aspartate-specific proteinase) protein and bcl-2 (B cell lymphoma/leukemia) protein in myocardium were detected to study the effect of LC intervention. From this study, it was found that the rats in control group have no obvious symptoms, while the rats in the intervention group have the same symptoms as the experimental group, but the symptoms were much lower in intensity than the experimental group. In the analysis of white blood cells in the 3 groups of rats, it was found that the white blood cells in the experimental group decreased significantly, followed by the intervention group, and there was no change in the control group. In the analysis of morphological changes of myocardial tissue in the 3 groups of rats, it is found that there was no significant change in myocardial cells in the control group, while patches of blood cells were found between myocardial tissues in the experimental group with nuclear deformation. There were blood spots between myocardial tissues in the intervention group, but there were no significant changes in nucleus. Among the changes in the content of TNF- $\alpha$ and apoptosis of myocardial tissue, it was found that the increase of TNF- $\alpha$ content and apoptotic phenomena were most obvious in the experimental group and there was a significant correlation between the experimental group and the control group. After LC intervention, it was found that the content of TNF- $\alpha$ in myocardial tissue is decreased and apoptotic phenomena are alleviated, which was significantly different from the experimental group. The expression changes of caspase- 3 and bcl-2 in myocardial tissue were detected by Western blotting. The results showed that the expression of caspase-3 and bcl-2 were the highest in the experimental group, followed by the intervention group and the lowest in the control group. In the analysis of the correlation between TNF- $\alpha$ and AI of myocardial cells, it is found that the changes in TNF- $\alpha$ is synchronous and positively correlated with the change of AI of myocardial cells. Therefore, through the above research, it is found that these results are basically consistent with the expected results before experiment.

In conclusion, by exploring the mechanism of LC intervention in rats with severe acute pancreatitis, it is found that LC intervention in severe acute pancreatitis could delay the apoptosis of myocardial cells in rats, which provided experimental data for the treatment of severe acute pancreatitis. However, there are still some deficiencies in this study, such as the small sample size in the experimental process, unable to accurately explore its mechanism. Therefore, it is necessary to increase the sample size to further explore its mechanism.

\section{REFERENCES}

1. Guadagni S, Cengeli I, Palmeri M. Early cholecystectomy for non-severe acute gallstone pancreatitis: easier said than done. Minerva Chir 2017;72(2):91-97.

2. Degrate L, Bernasconi D P, Meroni P. Mild acute biliary pancreatitis: the timing of cholecystectomy should not exceed index admission. Minerva Chir 2017;72(5):383-390.

3. Levenick JM, Gordon SR, Fadden LL. Rectal indomethacin does not prevent post-ERCP pancreatitis in consecutive patients. Gastroenterology 2016;150(4):911-917.

4. Khamaysi I, Singh P, Nasser S. The role of heparanase in the pathogenesis of acute pancreatitis: a potential therapeutic target. Sci Rep 2017;7(1):715.

5. Lv S, Fang Z, Wang A. One-Step LC and ERCP Treatment of 
40 Cases with Cholelithiasis Complicated with Common Bile Duct Stones. Hepato-Gastroenterol 2015;62(139):570-572.

6. Pinto LC, Rados DV, Barkan SS. Dipeptidyl peptidase-4 inhibitors, pancreatic cancer and acute pancreatitis: A metaanalysis with trial sequential analysis. Sci Rep 2018;8(1):782.

7. Yang L X, Liu J, Xing Y. Treating Moderately Severe Acute Pancreatitis with Raw Rhubarbs by Intranasal Jejuna Injection: a Randomized Clinical Analysis. Chin J Integr Med 2017;37(3):306-309.

8. Yang Q, Sun J, Chen Y Q. Multi-dimensional, comprehensive sample extraction combined with LC-GC/MS analysis for complex biological samples: application in the metabolomics study of acute pancreatitis. RSC Adv 2016;6(31):25837-25849.

9. Aksoy F, Demiral G, Ekinci Ö. Can the timing of laparoscopic cholecystectomy after biliary pancreatitis change the conversion rate to open surgery. Asian J Surg 2018;41(4):307312.

10. Bernas AA, Aveiga DA V, Etxeberria LZ. Acute pancreatitis in ICU secondary to treatment with tigecycline. Rev Esp Anestesiol Reanim 2017;64(1):46-49.

11. $\mathrm{Yu} \mathrm{H}, \mathrm{Chan} \mathrm{EEH}$, Lingam P. Index admission laparoscopic cholecystectomy for acute cholecystitis restores Gastrointestinal Quality of Life Index (GIQLI) score. J Hepatobiliary Pancreat Surg 2018;22(1):58-65.
12. Chyzhyk V, Kozmic S, Brown AS. Extreme hypertriglyceridemia: Genetic diversity, pancreatitis, pregnancy, and prevalence. J Clin Lipidol 2019;13(1):89-99.

13. Zerboni G, Capurso G, Di Pietropaolo M. The prevalence of pancreatic cystic lesions in patients with liver cirrhosis is double that in controls. United Eur Gastroent 2017;5(7):10071014.

14. Kiss L, Für G, Mátrai P. The effect of serum triglyceride concentration on the outcome of acute pancreatitis: systematic review and meta-analysis. Sci Rep 2018;8(1):14096.

15. Lam E T, Eckhardt S G, Messersmith W. Phase I study of enavatuzumab, a first-in-class humanized monoclonal antibody targeting the TWEAK receptor, in patients with advanced solid tumors. Mol Cancer Ther, 2018;17(1):215-221.

This is an open access article distributed under the terms of the Creative Commons Attribution-NonCommercial-ShareAlike 3.0 License, which allows others to remix, tweak, and build upon the work non-commercially, as long as the author is credited and the new creations are licensed under the identical terms

\begin{tabular}{l}
\hline This article was originally published in Special \\
issue on "Trends in therapeutic Management of \\
Various Conditions" Indian J Pharm Sci 2020:82(3) \\
spl issue 6;xx-xx
\end{tabular}

